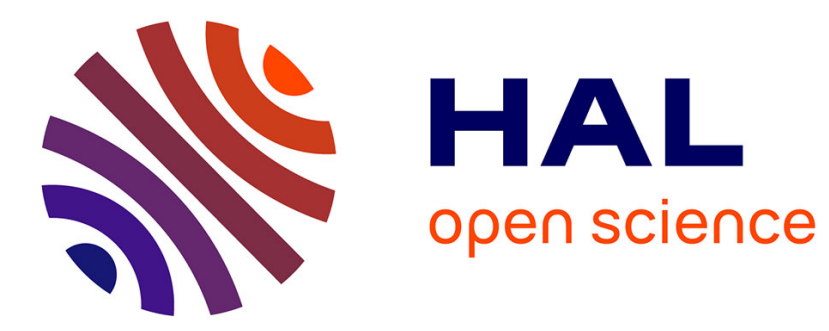

\title{
A Magnetic Vector Potential Volume Integral Formulation for Nonlinear Magnetostatic Problems
}

Vinh Le Van, Gérard Meunier, Olivier Chadebec, Jean-Michel Guichon

\section{To cite this version:}

Vinh Le Van, Gérard Meunier, Olivier Chadebec, Jean-Michel Guichon. A Magnetic Vector Potential Volume Integral Formulation for Nonlinear Magnetostatic Problems. IEEE Transactions on Magnetics, 2016, 52 (3), 10.1109/TMAG.2015.2490627 . hal-01385525

\section{HAL Id: hal-01385525 \\ https://hal.science/hal-01385525}

Submitted on 9 Nov 2020

HAL is a multi-disciplinary open access archive for the deposit and dissemination of scientific research documents, whether they are published or not. The documents may come from teaching and research institutions in France or abroad, or from public or private research centers.
L'archive ouverte pluridisciplinaire HAL, est destinée au dépôt et à la diffusion de documents scientifiques de niveau recherche, publiés ou non, émanant des établissements d'enseignement et de recherche français ou étrangers, des laboratoires publics ou privés. 


\title{
A Magnetic Vector Potential Volume Integral Formulation for Nonlinear Magnetostatic Problems
}

\author{
Vinh Le-Van, Gérard Meunier, Olivier Chadebec, and Jean-Michel Guichon \\ Grenoble Electrical Engineering Laboratory, Université Grenoble Alpes, Grenoble F-38000, France \\ Grenoble Electrical Engineering Laboratory, Centre National de la Recherche Scientifique, Grenoble F-38000, France
}

\begin{abstract}
Volume integral method (VIM) has been known as an interesting alternative to the finite-element method for electromagnetic field computation. Since only the active regions have to be meshed, VIM is very efficient for modeling of electromagnetic devices containing a predominated air volume. It is also adapted for multistatic studies with motion or optimization strategies. The aim of this paper is to propose a magnetic vector potential volume integral formulation in order to deal with the 3-D nonlinear magnetostatic problems. The main advantage of this formulation is that the convergence of nonlinear material solution can be easily reached after a few iterations without any relaxation. Moreover, the use of coarse meshes can lead to accurate results. Computed results for the TEAM Workshop problem 13 and for an actuator are reported.
\end{abstract}

Index Terms - Edge elements, integral equation, magnetic vector potential, nonlinear magnetostatic, team Workshop problem 13, volume integral method.

\section{INTRODUCTION}

$\mathbf{V}$ OLUME integral method (VIM) is known today as an interesting alternative to the classical finite-element method (FEM) for solving nonlinear magnetostatic problems. The main advantage of VIM versus FEM is that neither free space mesh nor boundary condition is required, only active regions have to be meshed. Associated with fast algorithms such as the fast multipole method (FMM) or hybrid cross approximation, the fully populated matrices system obtained by VIM can be compressed and treated rapidly.

To this day, several magnetostatic volume integral formulations have already been introduced [1]-[8]. The magnetic moment method (MMM) [1]-[3], which is the oldest and also the most popular one, is based on uniform magnetization on each element. This method is straightforward to implement and well adapted for simple geometry devices. However, it leads to numerical instability with the presence of nonlinear magnetic materials. In addition, the MMM is also strongly limited by the size of the obtained matrix (three unknowns per element). $\mathbf{H}$-edge, and $\phi$-nodal formulations were proposed in [4]-[7]. They seem to be more flexible and robust than the MMM for the treatment of complex devices. In general, both formulations present a quite poor convergence rate for nonlinear solutions due to the use of a $\mathbf{B}(\mathbf{H})$ curve. Moreover, the use of these formulations in order to model the multiply connected regions requires cutting techniques. Most recently, the B-facet formulation based on interpolation of magnetic flux on facet elements associated with an equivalent circuit approach has been proposed in [8]. This formulation ensures a good convergence rate due to the use of a $\mathbf{H}(\mathbf{B})$ curve. Its results present an excellent accuracy even with a very coarse mesh. Multiply connected regions were

Manuscript received July 6, 2015; revised September 15, 2015; accepted October 5, 2015. Date of publication October 14, 2015. treated straightforwardly, and the nonlinear problems convergence was easily reached without any relaxation technique. Nevertheless, the B-facet formulation requires independent loops search in order to restore the flux solenoidality. This task can be time consuming and decreases the convergence rate of linear systems solutions.

In this paper, an original magnetostatic volume integral formulation based on the interpolation of the magnetic vector potential on edge elements is proposed. This novel approach, called A-edge formulation, does not require the independent loops search algorithms. The formulation being auto-gauged, no gauge condition has to be added and the convergence rate of the linear solution is then improved. In others words, this new formulation has all the benefits of the $\mathbf{B}$-facet one but without its drawbacks.

\section{Magnetic Vector Potential Formulation}

Let us consider a 3-D magnetostatic problem composed by ferromagnetic regions $\Omega$ and coils with DC current density $\mathbf{J}$. We note $\Gamma$ the boundary between $\Omega$ and the free space.

\section{A. Basis Equations}

From Maxwell's equations, the governing equations for magnetostatics can be given in terms of magnetic flux density $\mathbf{B}$, magnetic field strength $\mathbf{H}$, and $\mathbf{J}$

$$
\begin{aligned}
\operatorname{div} \mathbf{B} & =0 \\
\operatorname{curl} \mathbf{H} & =\mathbf{J} .
\end{aligned}
$$

These equations are associated with constitutive laws

$$
\begin{aligned}
\mathbf{H} & =v(\mathrm{~B}) \mathrm{B} \\
\mathbf{M} & =\left(v_{0}-v(\mathrm{~B})\right) \mathrm{B}
\end{aligned}
$$

where $\mathbf{M}$ is the magnetization; $\boldsymbol{v}_{0}$ and $v(\mathbf{B})$ are the reluctivity of air and of ferromagnetic materials, respectively.

The decomposition of the magnetic field strength into the sum of the source magnetic field $\mathbf{H}_{0}$ and reduced magnetic 


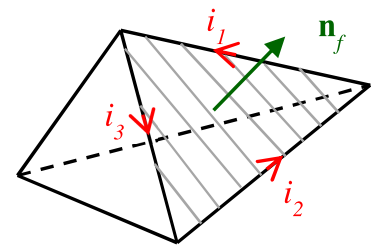

Fig. 1. Edge elements.

field $\mathbf{H}_{r}$ leads to write [8]

$$
v(\mathbf{B}) \mathbf{B}+\operatorname{grad} \varphi_{r}=\mathbf{H}_{0}
$$

where $\varphi_{r}$ is the reduced magnetic scalar potential, determined by [9]

$$
\varphi_{r}=\frac{1}{4 \pi} \int_{\Omega} \mathbf{M} \cdot \operatorname{grad}\left(\frac{1}{r}\right) d \Omega .
$$

By introducing the magnetic vector potential $\mathbf{A}$ that guarantees $\mathbf{B}=\mathbf{c u r l} \mathbf{A}$, (5) can be written as

$$
v(\text { B }) \operatorname{curl} \mathbf{A}+\operatorname{grad} \varphi_{r}=\mathbf{H}_{0} .
$$

From (4) and (6), the reduced scalar potential can be expressed as

$$
\varphi_{r}=\frac{1}{4 \pi} \int_{\Omega}\left(v_{0}-v(\mathbf{B})\right) \operatorname{curl} \mathbf{A} \cdot \operatorname{grad}\left(\frac{1}{r}\right) d \Omega .
$$

\section{B. Edge Elements Interpolation}

The magnetic vector potential $\mathbf{A}$ can be approximated by the first-order edge elements interpolation as

$$
\mathbf{A}=\sum_{j=1}^{N_{e}} \mathbf{w}_{j} A_{j}
$$

where $\mathbf{w}_{j}$ is the shape function of edge element $j, A_{j}$ the tangent component of vector potential on this edge, and $N_{e}$ is the number of edge elements in the ferromagnetic regions mesh. For an edge element $i$ belonging to the facet $f$ (Fig. 1), its first-order shape function satisfies [10]

$$
\operatorname{curl} \mathbf{w}_{i} \cdot \mathbf{n}_{f}=\operatorname{curl} w_{\text {in }}=\frac{1}{S_{f}}
$$

where $\mathbf{n}_{f}$ and $S_{f}$ are the normal vector and the surface of the facet $f$, respectively.

\section{Integral Formulations}

The projection of (7) into ferromagnetic domain $\Omega$ by the Galerkin method with the test functions curlw $i$ leads to

$$
\begin{aligned}
\int_{\Omega} \operatorname{curl} \mathbf{w}_{i} \cdot v(\mathbf{B}) \operatorname{curl} A d \Omega+ & \int_{\Omega} \operatorname{curl} \mathbf{w}_{i} \cdot \operatorname{grad} \varphi_{\text {red }} d \Omega \\
& =\int_{\Omega} \operatorname{curlw}_{i} \cdot \mathbf{H}_{0} d \Omega
\end{aligned}
$$

The two terms on the left hand can be developed as follows:

$$
\begin{aligned}
\int_{\Omega} \operatorname{curl} \mathbf{w}_{i} & \cdot v(\mathbf{B}) \operatorname{curl} \mathbf{A} d \Omega \\
= & \sum_{j=1}^{N_{e}}\left(\int_{\Omega} \operatorname{curl} \mathbf{w}_{i} \cdot v(\mathbf{B}) \operatorname{curl} \mathbf{w}_{j} d \Omega\right) A_{j}
\end{aligned}
$$
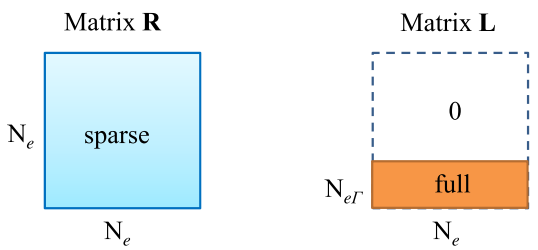

Fig. 2. Dimension of matrices $\mathbf{R}$ and $\mathbf{L}$.

$$
\begin{aligned}
& \int_{\Omega} \operatorname{curl} \mathbf{w}_{i} \cdot \operatorname{grad} \varphi_{\mathrm{red}} d \Omega \\
& =\int_{\Omega} \operatorname{div}\left(\operatorname{curl} \mathbf{w}_{i} \cdot \varphi_{r}\right) d \Omega=\int_{\Gamma} \operatorname{curl} w_{\mathrm{in}} \varphi_{r} d \Gamma \\
& =\int_{\Gamma} \operatorname{curl} w_{\mathrm{in}}\left(\frac{1}{4 \pi} \int_{\Omega}\left(v_{0}-v(\mathbf{B})\right) \operatorname{curl} \mathbf{A} \cdot \operatorname{grad}\left(\frac{1}{r}\right) d \Omega\right) d \Gamma .
\end{aligned}
$$

Since edge elements of first-order are used, the magnetic field $\mathbf{B}$ is constant in each tetrahedral element and nearly constant in each hexahedral element. Therefore, the reluctivity is constant in each volume element and (13) is transformed as

$$
\begin{aligned}
& \int_{\Omega} \operatorname{curl} \mathbf{w}_{i} \cdot \operatorname{grad} \varphi_{\text {red }} d \Omega \\
& =\int_{\Gamma} \operatorname{curl} w_{\text {in }}\left(\frac{1}{4 \pi} \sum_{j=1}^{N_{e}}\left(\sum_{f=1}^{N_{f}} \delta v_{f} \int_{f} \frac{\operatorname{curl} w_{j n}}{r} d f\right) A_{j}\right) d \Gamma
\end{aligned}
$$

where $N_{f}$ is the number of facets in the mesh and $\delta v_{f}$ is the jump of reluctivity between two adjacent elements sharing the facet $f$ or between the reluctivity an element belonging to the boundary and with the reluctivity of the air.

With (12) and (14), (11) can be written as

$$
([\mathbf{R}]+[\mathbf{L}])\{A\}=\left\{\mathbf{U}_{0}\right\} .
$$

The matrices $\mathbf{R}, \mathbf{L}$, and the vector $U_{0}$ are determined by

$$
\begin{aligned}
\mathbf{R}_{i j} & =\int_{\Omega} \operatorname{curlw}_{i} \cdot v(\mathbf{B}) \operatorname{curlw}_{j} d \Omega \\
\mathbf{L}_{i j} & =\frac{1}{4 \pi} \int_{\Gamma} \operatorname{curl} w_{\text {in }}\left(\sum_{f=1}^{N_{f}} \delta v_{f} \operatorname{curl} w_{j n} \int_{f} \frac{1}{r} d f\right) d \Gamma \\
\mathbf{U}_{0 i} & =\int_{\Omega} \operatorname{curlw}_{i} \cdot \mathbf{H}_{0} d \Omega .
\end{aligned}
$$

The dimension of sparse matrix $\mathbf{R}$ is $N_{e} \times N_{e}$ and one of the fully populated matrix $\mathbf{L}$ being $N_{e \Gamma} \times N_{e}$ with $N_{e \Gamma}$ is the number of edge elements on the boundary $\Gamma$. Their dimension is shown in Fig. 2. In general, $N_{e} \Gamma \ll N_{e}$.

Since (15) is autogauged, using an iterative solver such as GMRES (Generalized Minimal Residual Method) can solve it straightforwardly without any gauge condition requirement [11]. If the Newton-Raphson (NR) method is used in order to deal with nonlinear system, the integral matrix $\mathbf{L}$ should be computed only once before the resolution and then be updated at each iteration with a new $\delta v$. Note that the singularity of surface integral of Green's function can be analytically computed [12]. 


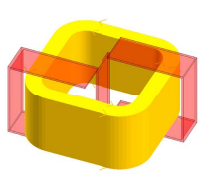

a)

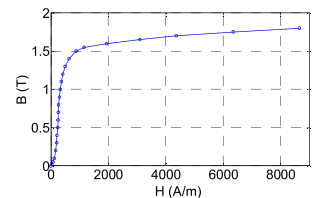

b)

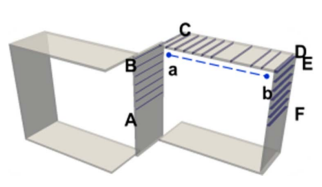

c)
Fig. 3. Description of the problem 13. (a) Device geometry. (b) $B-H$ curve of steel. (c) Specified positions for the magnetic field computation.

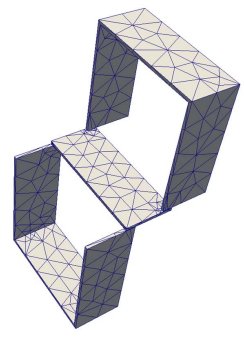

a)

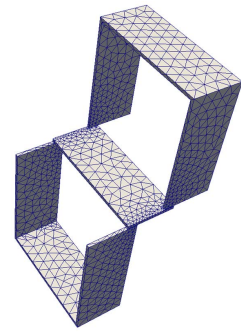

b)

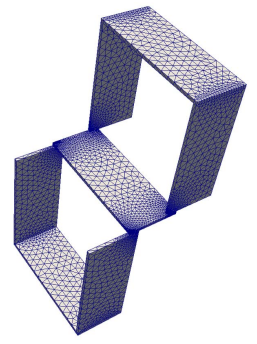

c)
Fig. 4. Meshes of steel plates of the problem 13. (a) 1235 elements. (b) 4855 elements. (c) 20737 elements.

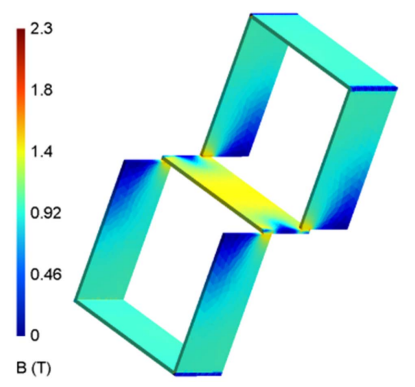

a)

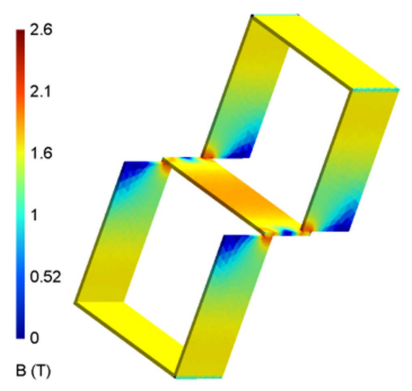

b)
Fig. 5. Magnetic flux density in steel plates. (a) 1000 AT. (b) 3000 AT.

\section{Applications AND Numerical Results}

\section{A. T.E.A.M. Workshop Problem 13}

Benchmark problem 13 defined in the T.E.A.M. Workshop is a 3-D nonlinear magnetostatic model. A coil excited by DC current is set between two steel channels, and a steel plate is inserted between the channels. The ampere turns are 1000 and 3000 AT which is sufficient to saturate the steel. The aim of this problem is to calculate the magnetic fields at various positions [13], [14]. Its description is summarized in Fig. 3.

Three meshes composed by 1235,4855 and 20738 tetrahedral elements (Fig. 4) were considered. The distribution of magnetic flux density in steel plates are shown in Fig. 5 corresponding with the mesh of 20737 elements.

The average magnetic flux density in steel plates (sections A-B-C-D-E-F) and magnetic flux density in the air (segment a-b) are shown in Figs. 6 and 7, respectively. For both ampere turns, the numerical results are very close to the measurement. A coarse mesh of 1235 elements was able to give reasonable results. Since the results obtained with the mesh of 4855 elements and with the one of 20737 elements

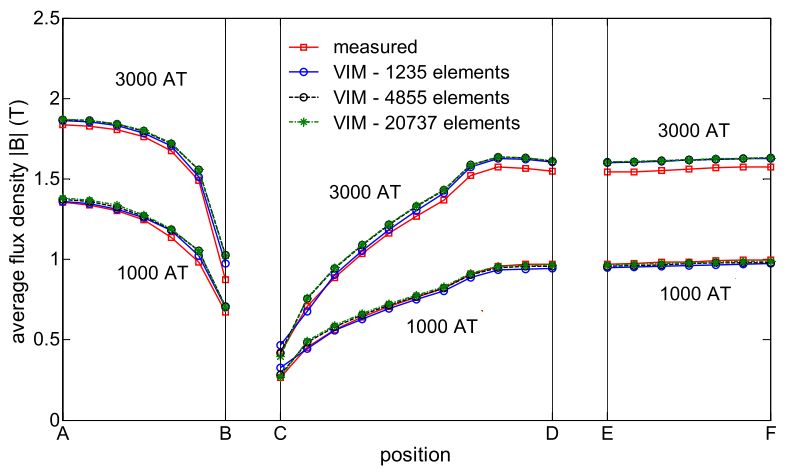

Fig. 6. Spatial distribution of average magnetic flux density in steel plates.

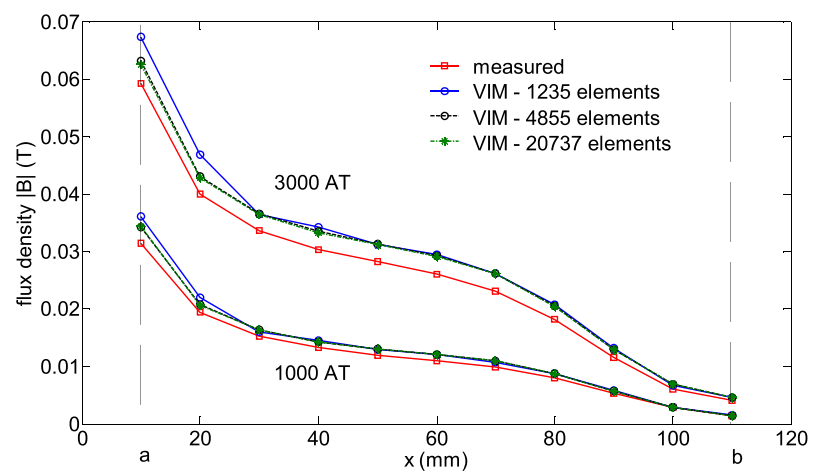

Fig. 7. Spatial distribution of magnetic flux density in air.

TABLE I

Computational Cost of Team Problem 13 Solution

\begin{tabular}{|c|c|c|c|c|c|c|}
\hline Mesh & \multirow{2}{*}{$\begin{array}{c}\text { Number } \\
\text { of edges }\end{array}$} & \begin{tabular}{c} 
Required \\
memory \\
\cline { 4 - 7 }
\end{tabular} & & \multicolumn{2}{|c|}{1000 AT } & \multicolumn{2}{|c|}{3000 AT } \\
\hline 1,235 & 2,180 & 21 & 5 & 7 & 13 & 16 \\
\hline 4,855 & 8,139 & 304 & 5 & 55 & 13 & 98 \\
\hline 20,737 & 33,697 & $213^{(*)}$ & 5 & $5,104^{(*)}$ & 13 & $7,786^{(*)}$ \\
\hline
\end{tabular}

CPU Intel Core i7-2600 3.4 GHz, OS 32 bits, 3GB of RAM.

${ }^{(*)}$ : times corresponding with integral matrix compressed by the FMM.

were superposed, it can be concluded that the convergence of solution was reached using the mesh with 4855 elements.

The discrepancies between the numerical results and the measurement at 3000 AT for the distribution of absolute value of flux density in air are larger than those at 1000 AT. However, the same difference was reported in [13].

The NR method was used for the resolution of nonlinear system with an absolute criterion $10^{-7}$. The linear system is solved by GMRES method using the incomplete LU decomposition of $\mathbf{R}$ as the preconditioner. Computational cost is reported in Table I.

We notice that the use of $\mathbf{B}$-facet formulation [8] leads to the same results as the A-edge one. However, the A-edge formulation is preferred in terms of linear system resolution. In effect, for this formulation, GMRES solver converged after 220 iterations without any preconditioning technique and computational time was $103 \mathrm{~s}$. In the same conditions, it took 580 iterations and $240 \mathrm{~s}$ for B-facet formulation. 


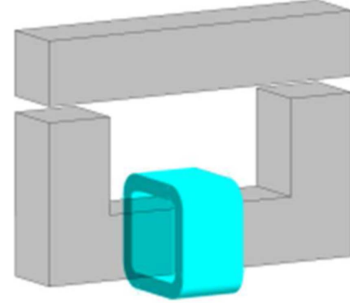

a)
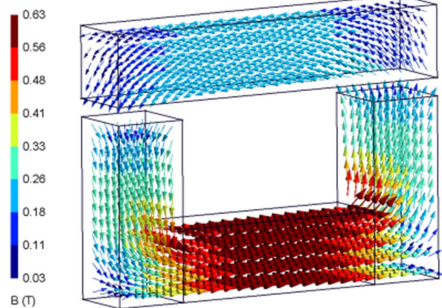

b)
Fig. 8. Actuator. (a) Geometry view. (b) Magnetic flux density distribution.

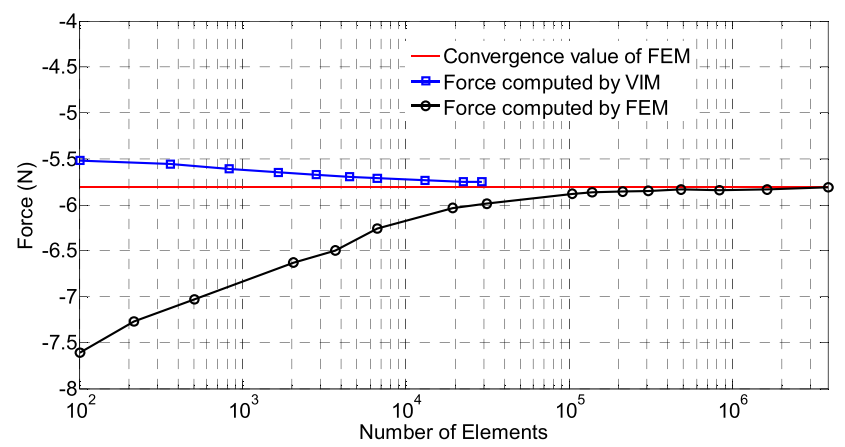

Fig. 9. Convergence of force computation.

This difference can be explained by the fact that the A formulation resolves the original linear equation system whereas the $\mathbf{B}$ formulation has to deal with a linear equations system depending the choice of independent loops [8], [15].

\section{B. Global Force Computation of an Actuator}

The proposed formulation was applied to model an actuator excited by a dc current coil [Fig. 8(a)]. The nonlinear material is approximated by an arctan law as reported in [8] with $J_{\mathrm{s}}=1 \mathrm{~T}$ and $\mu_{r}=1000$. The aim of this example is to compute the global force acting on the mobile part of the actuator.

The distribution of the magnetic flux density in the material is shown in Fig. 8(b). The global force was computed from the solution of the proposed formulation because of the equivalent magnetic charge method [16]. The convergence of the global force is given in Fig. 9. Compared with the results obtained by FEM with FLUX software, the convergence rate obtained with VIM is quite faster than FEM. With a mesh of 100 tetrahedral elements, the relative error of the computed force given by VIM is $\sim 5 \%$, the computation time being $3 \mathrm{~s}$. To reach the same results, FEM has to use a mesh composed by more than 10000 elements, the computation time being more than $1 \mathrm{~min}$.

\section{CONCLUSION}

An original integral volume formulation for solving nonlinear magnetostatic problems has been presented. This formulation is based on the interpolation of the magnetic vector potential on edge elements. The obtained equation system is autogauged and can be solved by an iterative solver without the imposition of a gauge condition. The singularity of Green's function can be analytically computed. The convergence of nonlinear solution is easily reached without any relaxation technique associated.

In general, the proposed formulation gives accurate results with a small computational cost. Even when coarse meshes were used, the obtained results remain acceptable. The formulation can be straightforwardly associated with fast algorithms in order to solving complex problems.

\section{REFERENCES}

[1] R. F. Harrington, Field Computation by Moment Methods. New York, NY, USA: Oxford Univ. Press, 1993.

[2] O. Chadebec, J.-L. Coulomb, and F. Janet, "A review of magnetostatic moment method," IEEE Trans. Magn., vol. 42, no. 4, pp. 515-520, Apr. 2006.

[3] A. Morandi, M. Fabbri, and P. L. Ribani, "A modified formulation of the volume integral equations method for 3-D magnetostatics," IEEE Trans. Magn., vol. 46, no. 11, pp. 3848-3859, Nov. 2010.

[4] L. Kettunen and L. Turner, "A volume integral formulation for nonlinear magnetostatics and eddy currents using edge elements," IEEE Trans. Magn., vol. 28, no. 2, pp. 1639-1642, Mar. 1992.

[5] A. Canova and M. Repetto, "Integral solution of nonlinear magnetostatic field problems," IEEE Trans. Magn., vol. 37, no. 3, pp. 1070-1077, May 2001.

[6] L. Han, L.-S. Tong, and J. Yang, "Integral equation method using total scalar potential for the simulation of linear or nonlinear 3D magnetostatic field with open boundary," IEEE Trans. Magn., vol. 30, no. 5, pp. 2897-2900, Sep. 1994.

[7] V. Le-Van, B. Bannwarth, A. Carpentier, O. Chadebec, J.-M. Guichon, and G. Meunier, "The adaptive cross approximation technique for a volume integral equation method applied to nonlinear magnetostatic problems," IEEE Trans. Magn., vol. 50, no. 2, Feb. 2014, Art. ID 7010904.

[8] V. Le-Van, G. Meunier, O. Chadebec, and J.-M. Guichon, "A volume integral formulation based on facet elements for nonlinear magnetostatic problems," IEEE Trans. Magn., vol. 51, no. 7, Jul. 2015, Art. ID 7002206.

[9] É. Durand, Magnétostatique. Paris, France: Masson et Cie, 1968.

[10] A. Bossavit, "Whitney forms: A class of finite elements for threedimensional computations in electromagnetism," IEE Proc. A, Phys. Sci., Meas. Instrum., Manage. Edu.-Rev., vol. 135, no. 8, pp. 493-500, Nov. 1988.

[11] Z. Ren, "Influence of the RHS on the convergence behaviour of the curl-curl equation," IEEE Trans. Magn., vol. 32, no. 3, pp. 655-658, May 1996.

[12] C. Rubeck, J. Yonnet, H. Allag, B. Delinchant, and O. Chadebec, "Analytical calculation of magnet systems: Magnetic field created by charged triangles and polyhedra," IEEE Trans. Magn., vol. 49, no. 1, pp. 144-147, Jan. 2013.

[13] T. Nakata and K. Fujiwara, "Summary of results for benchmark problem 13 (3-D nonlinear magnetostatic model)," COMPEL-Int. J. Comput. Math. Elect. Electron. Eng., vol. 11, no. 3, pp. 345-369, 1992.

[14] T. Nakata, N. Takahashi, and K. Fujiwara, "Summary of results for team workshop problem 13 (3-D nonlinear magnetostatic model)," COMPEL-Int. J. Comput. Math. Elect. Electron. Eng., vol. 14, nos. 2-3, pp. 91-101, 1995.

[15] G. Meunier et al., "A-T volume integral formulation for solving electromagnetic problems in the frequency domain," IEEE Trans. Magn., to be published. DOI: 10.1109/TMAG.2015.2496243

[16] H. L. Rakotoarison, V. Ardon, O. Chadebec, B. Delinchant, S. Guerin, and J. L. Coulomb, "Formal sensitivity computation of magnetic moment method," IEEE Trans. Magn., vol. 44, no. 6, pp. 1014-1017, Jan. 2008. 